\title{
Design, Synthesis and Biological Screening of Novel 1,5-Diphenyl-3-(4-(trifluoromethyl)phenyl)-2-pyrazoline Derivatives
}

\author{
Fatih Tok ${ }^{1}$ and Bedia Koçyiğit-Kaymakçığglu ${ }^{1, \star}$ \\ ${ }^{1}$ Department of Pharmaceutical Chemistry, Faculty of Pharmacy, Marmara University, Istanbul-34668, Turkey \\ * Corresponding author: E-mail: bkaymakcioglu@marmara.edu.tr \\ Tel: +90-216-4142963/1023
}

Received: 04-01-2020

\begin{abstract}
1-Phenyl-5-substituted-3-(4-(trifluoromethyl)phenyl)-4,5-dihydro- $1 H$-pyrazole derivatives were synthesized from chalcone derivatives. The structures of compounds were characterized by IR, ${ }^{1} \mathrm{H}$ NMR spectroscopic methods and elemental analysis. All compounds were evaluated for their in vitro antioxidant activity using DPPH and ABTS methods, anti-inflammatory activity using lipoxygenase inhibitory method and antidiabetic activity using the $\alpha$-glucosidase inhibitory method. Especially, pyrazoline derivatives exhibited stronger anti-inflammatory activity than the reference drug indomethacin $\left(\mathrm{IC}_{50}: 50.45 \mu \mathrm{M}\right)$ and their $\mathrm{IC}_{50}$ values were in the range of 0.68 and $4.45 \mu \mathrm{M}$. In addition, the ADME properties of all chalcone and pyrazoline derivatives were calculated by Lipinski's and Veber's rules.
\end{abstract}

Keywords: 2-Pyrazoline; lipoxygenase enzyme; $\alpha$-glucosidase; ABTS and DPPH

\section{Introduction}

Pyrazoline scaffolds bearing five-membered heterocyclic ring systems are used frequently in organic synthesis and medicinal chemistry because of their broad spectrum of activities. ${ }^{1}$ Pyrazoline rings have important pharmacological and biological properties such as antioxidant, anti-inflammatory, analgesic, antimicrobial, antimalarial, antihypertensive, anticonvulsant, antidepressant, anticancer. $^{2-6}$ We studied the synthesis and antiproliferative activity of some pyrazoline compounds in our previous study. ${ }^{7}$ Pyrazolines exhibited these different activities by interact- ing with some receptors or enzymes. For example, ElBordiny et al. demonstrated in their study that pyrazolines are superior lipoxygenase enzyme inhibitors compared to the reference drug. ${ }^{8}$ Furthermore, Chaudhry et al. and Sathish et al. reported pyrazoline derivatives as alpha-glucosidase inhibitors. ${ }^{9} 10$ Additionally, many studies proved different activities of pyrazoline derivatives as receptor tyrosine kinase, topoisomerase 1, carbonic anhydrase and cholinesterase inhibitors. It was found that nitrogen atoms of the pyrazoline ring and at least one substitution with aryl moiety are essential for anti-inflammatory activity (Gawad $e t$ al., 2012). ${ }^{11-15}$
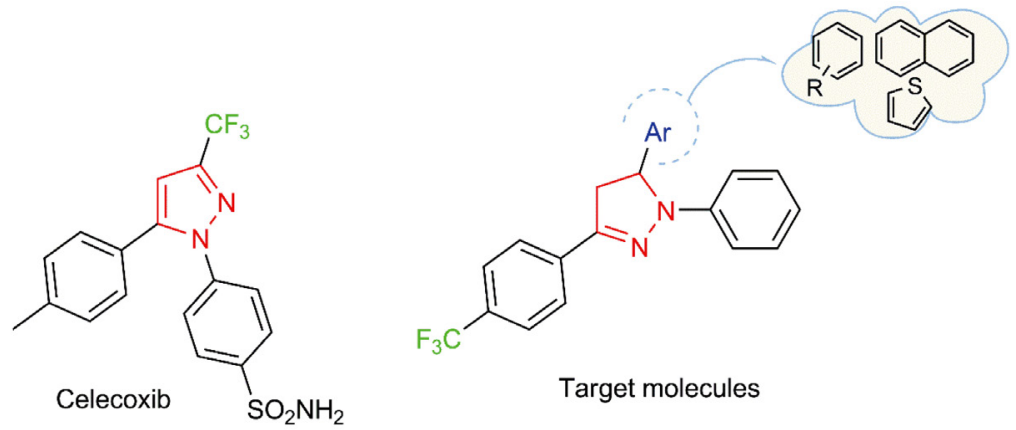

Figure 1. Chemical structure of designed compounds and celecoxib 
Several drug molecules carrying pyrazole core with different activities are currently available in the market, for example, antipyrine (analgesic), celecoxib (anti-inflammatory), pyrazofurin (antibiotic). ${ }^{16}$

It is known that fluorine substitution increases biological activity and metabolic-chemical stability (compared to $\mathrm{C}-\mathrm{H}$ bond) in drug research. Furthermore, fluorine alters physicochemical properties and enhances binding affinity to target protein easily. ${ }^{17-18}$ We designed new molecules which are carrying pyrazoline core and trifluoromethyl substitution on the aromatic ring (Figure 1).

In this study, we synthesized chalcone derivatives from 4'-(trifluoromethyl)acetophenone in the first step. Then we synthesized new 2-pyrazoline derivatives from chalcones. We aimed to show different biological activities of pyrazoline derivatives. Therefore all synthesized compounds were screened for their antioxidant activity using DPPH and ABTS method, anti-inflammatory activity using lipoxygenase inhibitory method, and antidiabetic activity using $\alpha$-glucosidase inhibitory methods.

\section{Experimental}

\section{1. Synthesis}

Chemicals and solvents were obtained from Sigma Aldrich (St. Louis, MO, USA) or Merck (Darmstadt, Germany). The progress of the reaction was monitored via thin layer chromatography (TLC), performed on commercially available silica gel (Kieselgel 60, F254) coated aluminum sheets (Merck) by using developing systems: petroleum ether/acetone $(60: 40 \mathrm{v} / \mathrm{v})$ as a solvent system. The visualization on TLC was done under ultraviolet (UV) light $(\lambda=254 \mathrm{~nm})$. Melting points were determined by Schmelzpunktbestimmer SMP II apparatus. Infrared spectra were recorded on a Shimadzu FTIR $8400 \mathrm{~S}$ Spectrometer and data was expressed in wavenumber $v\left(\mathrm{~cm}^{-1}\right)$. Proton nuclear magnetic resonance (NMR) $(400 \mathrm{MHz})$ spectra were recorded with a Bruker ACP 200 spectrometer (Bruker Corp., Billerica, MA, USA). Deuterated dimethylsulfoxide (DMSO- $d_{6}$ ) was used as the solvent and tetramethylsilane (TMS) as the internal standard. The chemical shift values $(\delta)$ were expressed in ppm. Elemental analyses $(\mathrm{C}, \mathrm{H}$ and $\mathrm{N}$ ) was performed on a CHNS-Thermo Scientific Flash 2000 (Waltham, MA, USA).

\section{1. 1. General Procedure for the Synthesis of Chalcone}

$1 \mathrm{mmol}$ of 4'-(trifluoromethyl)acetophenone and equimolar quantities of substituted aromatic aldehydes were dissolved in methanol, then $\mathrm{NaOH}$ ( $50 \%$ water solution) was added to the reaction mixture. It was stirred at room temperature for $16 \mathrm{~h}$ and then poured into ice-cold water. The precipitated product was washed with water, filtered and recrystallized from methanol. ${ }^{19}$

\section{1. 2. General Procedure for the Synthesis of Pyrazoline}

$10 \mathrm{mmol}$ of chalcone derivatives, $10 \mathrm{mmol}$ of phenylhydrazine hydrochloride and $10 \mathrm{~mL}$ of glacial acetic acid were put in a reaction flask. The content was refluxed and stirred for $12 \mathrm{~h}$. Then, it was neutralized with dilute ammonia solution. The precipitate was washed with water, filtered and recrystallized from ethanol. ${ }^{20}$

\section{3-(2,6-Dimethylphenyl)-1-(4-(trifluoromethyl)phenyl)} prop-2-en-1-one (1a)

White powder, yield $77 \%, \mathrm{mp} 74.3-74.5^{\circ} \mathrm{C}$. IR (KBr) v 3064, 2978, 2912, 1696,1606, 1591, 1573, 1510, 1408, $1315,1213,1161,1064,835 \mathrm{~cm}^{-1} .{ }^{1} \mathrm{H}$ NMR $(400 \mathrm{MHz}$, DMSO- $\left.d_{6}\right) \delta 2.38\left(\mathrm{~s}, 6 \mathrm{H}, 2 \mathrm{CH}_{3}\right), 7.12-7.87(\mathrm{~m}, 5 \mathrm{H}, \mathrm{Ar}-\mathrm{H}$ and $\mathrm{CH}=\mathrm{CH}), 7.92(\mathrm{~d}, J=8.4 \mathrm{~Hz}, 2 \mathrm{H}, \mathrm{Ar}-\mathrm{H}) 8.27(\mathrm{~d}, J=$ $8.4 \mathrm{~Hz}, 2 \mathrm{H}, \mathrm{Ar}-\mathrm{H}$ ). Anal. Calcd for $\mathrm{C}_{18} \mathrm{H}_{15} \mathrm{~F}_{3} \mathrm{O}: \mathrm{C}, 71.04$; $\mathrm{H}$, 4.97. Found: $\mathrm{C}, 70.82 ; \mathrm{H}, 4.86 \%$.

3-(2,6-Dichlorophenyl)-1-(4-(trifluoromethyl)phenyl) prop-2-en-1-one (1b)

Yellow powder, yield $65 \%, \mathrm{mp} 62.3-62.7{ }^{\circ} \mathrm{C}$. IR $(\mathrm{KBr})$ v 3072, 1696, 1606, 1573, 1508, 1467, 1408, 1305, 1213, 1161, 1064, $835 \mathrm{~cm}^{-1} .{ }^{1} \mathrm{H}$ NMR $(400 \mathrm{MHz}, \mathrm{DM}-$ SO- $\left.d_{6}\right) \delta 7.41-8.24(\mathrm{~m}, 9 \mathrm{H}, \mathrm{Ar}-\mathrm{H}$ and $\mathrm{CH}=\mathrm{CH})$. Anal. Calcd for $\mathrm{C}_{16} \mathrm{H}_{9} \mathrm{Cl}_{2} \mathrm{~F}_{3} \mathrm{O}$ : C, 55.68; $\mathrm{H}, 2.63$. Found: C, 55.48; H, 2.78.

3-o-Tolyl-1-(4-(trifluoromethyl)phenyl)prop-2-en-1one (1c)

Yellow powder, yield $75 \%, \mathrm{mp} 89.0-89.8^{\circ} \mathrm{C}$. IR $(\mathrm{KBr})$ $v$ 3053, 2978, 2897, 1683, 1595, 1510, 1462, 1410, 1317, 1211, 1161, 1064, $833 \mathrm{~cm}^{-1} .{ }^{1} \mathrm{H}$ NMR $(400 \mathrm{MHz}, \mathrm{DM}-$ $\left.\mathrm{SO}-d_{6}\right) \delta 2.42(\mathrm{~s}, 3 \mathrm{H}, \mathrm{CH}), 7.26-8.32(\mathrm{~m}, 10 \mathrm{H}, \mathrm{Ar}-\mathrm{H}$ and $\mathrm{CH}=\mathrm{CH}$ ). Anal. Calcd for $\mathrm{C}_{17} \mathrm{H}_{13} \mathrm{~F}_{3} \mathrm{O}: \mathrm{C}, 70.34 ; \mathrm{H}, 4.51$. Found: C, 70.56; H, 4.55 .

3-(4-Isopropylphenyl)-1-(4-(trifluoromethyl)phenyl) prop-2-en-1-one (1d)

White powder, yield $81 \%, \mathrm{mp} 84.7-85.3^{\circ} \mathrm{C}$. IR (KBr) $v 3053,2960,2928,1660,1600,1579,1510,1408,1319$, $1215,1165,1066,821 \mathrm{~cm}^{-1} .{ }^{1} \mathrm{H}$ NMR $(600 \mathrm{MHz}, \mathrm{DM}-$ SO- $\left.d_{6}\right) \delta 1.23\left(\mathrm{~d}, J=7.2 \mathrm{~Hz}, 6 \mathrm{H}, \mathrm{CH}_{3}\right), 2.94(\mathrm{~m}, 1 \mathrm{H}, \mathrm{CH})$, 7.35-8.33 (m, 10H, Ar-H and $\mathrm{CH}=\mathrm{CH})$. Anal. Calcd for $\mathrm{C}_{19} \mathrm{H}_{17} \mathrm{~F}_{3} \mathrm{O}: \mathrm{C}, 71.69 ; \mathrm{H}, 5.38$. Found: $\mathrm{C}, 71.44 ; \mathrm{H}, 5.46$.

\footnotetext{
3-Phenyl-1-(4-(trifluoromethyl)phenyl)prop-2-en-1one (1e)

White powder, yield $75 \%, \mathrm{mp} 113.7-114.3{ }^{\circ} \mathrm{C}$. IR $(\mathrm{KBr})$ v 3063, 1664, 1600, 1573, 1510, 1410, 1317, 1217, $1159,1064,839 \mathrm{~cm}^{-1} .{ }^{1} \mathrm{H}$ NMR $\left(600 \mathrm{MHz}\right.$, DMSO- $\left.d_{6}\right) \delta$ 7.44-8.35 (m, 11H, Ar-H and $\mathrm{CH}=\mathrm{CH})$. Anal. Calcd for
} 
$\mathrm{C}_{16} \mathrm{H}_{11} \mathrm{~F}_{3} \mathrm{O}: \mathrm{C}, 69.56 ; \mathrm{H}, 4.01$. Found: $\mathrm{C}, 69.78 ; \mathrm{H}, 4.07$ (CAS Number: 62056-10-4). ${ }^{21}$

3-(4-Chlorophenyl)-1-(4-(trifluoromethyl)phenyl) prop-2-en-1-one (1f)

White powder, yield $69 \%, \mathrm{mp} 125.2-125.7^{\circ} \mathrm{C}$ (CAS Number: 57076-98-9). ${ }^{22}$

\section{3-p-Tolyl-1-(4-(trifluoromethyl)phenyl)prop-2-en-1- one (1g)}

White powder, yield $72 \%$, mp $147.8-148.4{ }^{\circ} \mathrm{C}$ (CAS Number: 1551606-21-3). ${ }^{23}$

\section{3-m-Tolyl-1-(4-(trifluoromethyl)phenyl)prop-2-en-1- one (1h)}

White powder, yield $82 \%$, mp $92.0-92.5^{\circ} \mathrm{C}$. IR $(\mathrm{KBr})$ v 3061, 2978, 2922, 1662, 1600,1573,1510,1450,1410, $1317,1217,1159,1064,837 \mathrm{~cm}^{-1} .{ }^{1} \mathrm{H}$ NMR $(400 \mathrm{MHz}$, DMSO- $\left.d_{6}\right) \delta 2.34(\mathrm{~s}, 3 \mathrm{H}, \mathrm{CH}), 7.26-8.31(\mathrm{~m}, 10 \mathrm{H}, \mathrm{Ar}-\mathrm{H}$ and $\mathrm{CH}=\mathrm{CH}$ ). Anal. Calcd for $\mathrm{C}_{17} \mathrm{H}_{13} \mathrm{~F}_{3} \mathrm{O}: \mathrm{C}, 70.34 ; \mathrm{H}$, 4.51. Found: C, 70.47; H, 4.44.

3-(Thiophen-2-yl)-1-(4-(trifluoromethyl)phenyl)prop2-en-1-one (1i)

White powder, yield $65 \%$, mp $135.4-135.9^{\circ} \mathrm{C}$ (CAS Number: $1372376-05-0){ }^{24}$

3-(4-(Dimethylamino)phenyl)-1-(4-(trifluoromethyl) phenyl)prop-2-en-1-one (1j)

White powder, yield $75 \%, \mathrm{mp} 140.5-141.2^{\circ} \mathrm{C}$ (CAS Number: 1940174-93-5). ${ }^{25}$

3-(Naphthalen-1-yl)-1-(4-(trifluoromethyl)phenyl) prop-2-en-1-one (1k)

Yellow powder, yield $74 \%, \mathrm{mp} 92.0-92.5^{\circ} \mathrm{C}$. IR $(\mathrm{KBr})$ $v$ 3078, 2980,1660,1593, 1573, 1435, 1408, 1319, 1215, $1163,1064,839 \mathrm{~cm}^{-1} .{ }^{1} \mathrm{H}$ NMR $\left(400 \mathrm{MHz}\right.$, DMSO- $\left.d_{6}\right) \delta$ 7.59-8.64 (m, $13 \mathrm{H}$, Ar- $\mathrm{H}$ and $\mathrm{CH}=\mathrm{CH})$. Anal. Calcd for $\mathrm{C}_{20} \mathrm{H}_{13} \mathrm{~F}_{3} \mathrm{O}: \mathrm{C}, 73.61 ; \mathrm{H}, 4.12$. Found: C, 73.87; H, 4.22.

5-(2,6-Dimethylphenyl)-1-phenyl-3-(4-(trifluoromethyl)phenyl)-4,5-dihydro-1 $H$-pyrazole (2a)

Yellow powder, yield $75 \%, \mathrm{mp} 133.3-133.8{ }^{\circ} \mathrm{C}$. IR $(\mathrm{KBr})$ v 3068, 2974, 2912, 1616, 1593, 1510, 1408, 1319, $1215,1163,1064,839 \mathrm{~cm}^{-1} .{ }^{1} \mathrm{H}$ NMR (300 MHz, DMSO- $d_{6}$ ) $\delta 2.04$ and $2.55\left(2 \mathrm{~s}, 6 \mathrm{H}, 2 \mathrm{CH}_{3}\right), 3.16(\mathrm{dd}, \mathrm{Jax}=6.33 \mathrm{~Hz}, \mathrm{Jab}=$ $17.88 \mathrm{~Hz}, 1 \mathrm{H}, \mathrm{Ha}), 4.02(\mathrm{dd}, J b x=13.75 \mathrm{~Hz}, J a b=17.88 \mathrm{~Hz}$, $1 \mathrm{H}, \mathrm{Hb}$ ), 5.75 (dd, Jax $=6.33 \mathrm{~Hz}, J b x=13.76 \mathrm{~Hz}, 1 \mathrm{H}, \mathrm{Hx}$ ), 6.72-7.17 (m, 8H, Ar-H), 7.77 (d, J= 8.1 Hz, 2H, Ar-H), 7.92 $\left(\mathrm{d}, J=8.1 \mathrm{~Hz}, 2 \mathrm{H}\right.$, Ar-H). Anal. Calcd for $\mathrm{C}_{24} \mathrm{H}_{21} \mathrm{~F}_{3} \mathrm{~N}_{2}: \mathrm{C}$, 73.08; H, 5.37; N, 7.10. Found: C, 73.55; H, 5.27; N, 7.35.

5-(2,6-Dichlorophenyl)-1-phenyl-3-(4-(trifluoromethyl)phenyl)-4,5-dihydro- $1 H$-pyrazole (2b)

Yellow powder, yield $65 \%, \mathrm{mp} 142.2-142.9{ }^{\circ} \mathrm{C}$. IR $(\mathrm{KBr}) \vee 3055,1618,1587,1521,1498,1321,1247,1118$,
1064, $839 \mathrm{~cm}^{-1} .{ }^{1} \mathrm{H}$ NMR $\left(400 \mathrm{MHz}, \mathrm{DMSO}-d_{6}\right) \delta 3.24$ $(\mathrm{dd}, J a x=6.12 \mathrm{~Hz}, J a b=17.44 \mathrm{~Hz}, 1 \mathrm{H}, \mathrm{Ha}), 3.96(\mathrm{dd}, J b x=$ $13.96 \mathrm{~Hz}, J a b=17.44 \mathrm{~Hz}, 1 \mathrm{H}, \mathrm{Hb}), 6.14(\mathrm{dd}, J a x=6.12 \mathrm{~Hz}$, $J b x=13.96 \mathrm{~Hz}, 1 \mathrm{H}, \mathrm{Hx}), 6.72-7.92(\mathrm{~m}, 12 \mathrm{H}, \mathrm{Ar}-\mathrm{H})$. Anal. Calcd for $\mathrm{C}_{22} \mathrm{H}_{15} \mathrm{Cl}_{2} \mathrm{~F}_{3} \mathrm{~N}_{2}: \mathrm{C}, 60.71 ; \mathrm{H}, 3.47 ; \mathrm{N}, 6.44$. Found: C, 60.22; H, 3.37; N, 6.66.

\section{1-Phenyl-5-o-tolyl-3-(4-(trifluoromethyl)phenyl)-} 4,5-dihydro- $1 \mathrm{H}$-pyrazole (2c)

Yellow powder, yield $77 \%, \mathrm{mp} 119.6-120.1{ }^{\circ} \mathrm{C}$. IR $(\mathrm{KBr})$ v 3059, 2980, 2916, 1616, 1587, 1521, 1495, 1319, $1217,1163,1064,839 \mathrm{~cm}^{-1} .{ }^{1} \mathrm{H}$ NMR $(400 \mathrm{MHz}, \mathrm{DM}-$ SO- $\left.d_{6}\right) \delta 2.43\left(\mathrm{~s}, 3 \mathrm{H}, \mathrm{CH}_{3}\right), 3.04(\mathrm{dd}, \mathrm{Jax}=6.55 \mathrm{~Hz}, \mathrm{Jab}=$ $17.52 \mathrm{~Hz}, 1 \mathrm{H}, \mathrm{Ha}), 3.99$ (dd, Jbx $=13.60 \mathrm{~Hz}, J a b=17.52$ $\mathrm{Hz}, 1 \mathrm{H}, \mathrm{Hb}), 5.64(\mathrm{dd}, J a x=6.56 \mathrm{~Hz}, J b x=13.60 \mathrm{~Hz}, 1 \mathrm{H}$, $\mathrm{Hx}), 6.72-7.25(\mathrm{~m}, 9 \mathrm{H}, \mathrm{Ar}-\mathrm{H}), 7.74(\mathrm{~d}, J=8.4 \mathrm{~Hz}, 2 \mathrm{H}, \mathrm{Ar}-$ $\mathrm{H}), 7.91(\mathrm{~d}, J=8.4 \mathrm{~Hz}, 2 \mathrm{H}, \mathrm{Ar}-\mathrm{H})$. Anal. Calcd for $\mathrm{C}_{23} \mathrm{H}-$ ${ }_{19} \mathrm{~F}_{3} \mathrm{~N}_{2}$ : C, 72.62; H, 5.03; N, 7.36. Found: C, 72.32; H, 5.17; $\mathrm{N}, 7.47$.

5-(4-Isopropylphenyl)-1-phenyl-3-(4-(trifluoromethyl) phenyl)-4,5-dihydro- $1 \mathrm{H}$-pyrazole (2d)

Orange powder, yield $81 \%$, mp $89.1-89.4{ }^{\circ} \mathrm{C}$. IR $(\mathrm{KBr})$ v 3051, 2960, 2899, 1618, 1595, 1556, 1498, 1319, 1249, 1163, 1064, $839 \mathrm{~cm}^{-1}$. ${ }^{1} \mathrm{H}$ NMR $(400 \mathrm{MHz}, \mathrm{DM}-$ SO- $\left.d_{6}\right) \delta 0.97$ and $1.55\left(2 \mathrm{~d}, 6 \mathrm{H}, 2 \mathrm{CH}_{3}\right), 2.79(\mathrm{~m}, 1 \mathrm{H}, \mathrm{CH})$, $3.14(\mathrm{dd}, \mathrm{Jax}=6.24 \mathrm{~Hz}, J a b=17.28 \mathrm{~Hz}, 1 \mathrm{H}, \mathrm{Ha}), 3.95(\mathrm{dd}$, $J b x=13.45 \mathrm{~Hz}, J a b=17.27 \mathrm{~Hz}, 1 \mathrm{H}, \mathrm{Hb}), 5.56(\mathrm{dd}, J a x=$ $6.24 \mathrm{~Hz}, J b x=13.44 \mathrm{~Hz}, 1 \mathrm{H}, \mathrm{Hx}), 6.72-7.52(\mathrm{~m}, 9 \mathrm{H}, \mathrm{Ar}-$ $\mathrm{H}), 7.73(\mathrm{~d}, J=8.1 \mathrm{~Hz}, 2 \mathrm{H}, \mathrm{Ar}-\mathrm{H}), 7.93(\mathrm{~d}, J=8.1 \mathrm{~Hz}, 2 \mathrm{H}$, Ar-H). Anal. Calcd for $\mathrm{C}_{25} \mathrm{H}_{23} \mathrm{~F}_{3} \mathrm{~N}_{2}$ : C, 73.51; H, 5.68; N, 6.86. Found: C, 73.77; H, 5.49; N, 6.75 .

1,5-Diphenyl-3-(4-(trifluoromethyl)phenyl)-4,5-dihydro- $1 H$ - pyrazole $(2 \mathrm{e})$

Yellow powder, yield $73 \%, \mathrm{mp} 108.2-108.4{ }^{\circ} \mathrm{C}$. IR $(\mathrm{KBr})$ v 3064, 1618, 1595, 1554, 1496, 1321, 1249, 1163 , 1064, $837 \mathrm{~cm}^{-1}$. ${ }^{1} \mathrm{H}$ NMR (600 MHz, DMSO- $\left.d_{6}\right) \delta 3.17$ $(\mathrm{dd}, J a x=6.66 \mathrm{~Hz}, J a b=17.46 \mathrm{~Hz}, 1 \mathrm{H}, \mathrm{Ha}), 3.96(\mathrm{dd}, J b x=$ $12.49 \mathrm{~Hz}, J a b=17.44 \mathrm{~Hz}, 1 \mathrm{H}, \mathrm{Hb}), 5.58(\mathrm{dd}, J a x=6.66 \mathrm{~Hz}$, $J b x=12.49 \mathrm{~Hz}, 1 \mathrm{H}, \mathrm{Hx}), 6.74-7.51(\mathrm{~m}, 10 \mathrm{H}, \mathrm{Ar}-\mathrm{H}), 7.78$ $(\mathrm{d}, J=8.4 \mathrm{~Hz}, 2 \mathrm{H}, \mathrm{Ar}-\mathrm{H}), 7.94(\mathrm{~d}, J=8.4 \mathrm{~Hz}, 2 \mathrm{H}, \mathrm{Ar}-\mathrm{H})$. Anal. Calcd for $\mathrm{C}_{22} \mathrm{H}_{17} \mathrm{~F}_{3} \mathrm{~N}_{2}$ : C, 72.12; H, 4.68; N, 7.65. Found: C, 72.31; H, 4.63; N, 7.77.

5-(4-Chlorophenyl)-1-phenyl-3-(4-(trifluoromethyl) phenyl)-4,5-dihydro- $1 \mathrm{H}$-pyrazole (2f)

Orange powder, yield $66 \%, \mathrm{mp} 79.1-79.3{ }^{\circ} \mathrm{C}$. IR $(\mathrm{KBr})$ v 3055, 1618, 1597, 1554, 1496, 1319, 1249, 1163, 1064, $839 \mathrm{~cm}^{-1} .{ }^{1} \mathrm{H}$ NMR (400 MHz, DMSO- $\left.d_{6}\right) \delta 3.16$ $(\mathrm{dd}, J a x=6.24 \mathrm{~Hz}, J a b=17.28 \mathrm{~Hz}, 1 \mathrm{H}, \mathrm{Ha}$ ), 3.96 (dd, Jbx = $12.40 \mathrm{~Hz}, J a b=17.28 \mathrm{~Hz}, 1 \mathrm{H}, \mathrm{Hb}), 5.60(\mathrm{dd}, \mathrm{Jax}=6.23 \mathrm{~Hz}$, $J b x=12.41 \mathrm{~Hz}, 1 \mathrm{H}, \mathrm{Hx}), 6.74-7.51(\mathrm{~m}, 9 \mathrm{H}, \mathrm{Ar}-\mathrm{H}), 7.75(\mathrm{~d}$, $J=8.4 \mathrm{~Hz}, 2 \mathrm{H}, \mathrm{Ar}-\mathrm{H}), 7.91(\mathrm{~d}, J=8.4 \mathrm{~Hz}, 2 \mathrm{H}, \mathrm{Ar}-\mathrm{H})$. Anal. Calcd for $\mathrm{C}_{22} \mathrm{H}_{16} \mathrm{ClF}_{3} \mathrm{~N}_{2}$ : C, 65.92; H, 4.02; N, 6.99. Found: C, $65.75 ; \mathrm{H}, 3.88 ; \mathrm{N}, 6.72$. 
1-Phenyl-5-p-tolyl-3-(4-(trifluoromethyl)phenyl)- 4,5dihydro- $1 H$-pyrazole $(2 \mathrm{~g})$

Yellow powder, yield $80 \%, \mathrm{mp} 123.4-123.7^{\circ} \mathrm{C}$. IR $(\mathrm{KBr}) \vee 3007,2924,2852,1614,1597,1552,1498,1321$, $1244,1168,1064,835 \mathrm{~cm}^{-1} .{ }^{1} \mathrm{H}$ NMR $\left(300 \mathrm{MHz}, \mathrm{DMSO}-d_{6}\right)$ $\delta 2.25\left(\mathrm{~s}, 3 \mathrm{H}, \mathrm{CH}_{3}\right), 3.16(\mathrm{dd}, J a x=6.30 \mathrm{~Hz}, J a b=17.91 \mathrm{~Hz}$, $1 \mathrm{H}, \mathrm{Ha}$ ), 3.93 (dd, Jbx = 12.42 Hz, Jab = 17.91 Hz, 1H, Hb), $5.56(\mathrm{dd}, J a x=6.31 \mathrm{~Hz}, J b x=12.40 \mathrm{~Hz}, 1 \mathrm{H}, \mathrm{Hx}), 6.72-7.19$ (m, 9H, Ar-H), 7.77 (d, $J=8.1 \mathrm{~Hz}, 2 \mathrm{H}, \mathrm{Ar}-\mathrm{H}), 7.94(\mathrm{~d}, J=$ $8.1 \mathrm{~Hz}, 2 \mathrm{H}, \mathrm{Ar}-\mathrm{H})$. Anal. Calcd for $\mathrm{C}_{23} \mathrm{H}_{19} \mathrm{~F}_{3} \mathrm{~N}_{2}: \mathrm{C}, 72.62 ; \mathrm{H}$, 5.03; N, 7.36. Found: C, 72.35; H, 5.20; N, 7.57.

1-Phenyl-5-m-tolyl-3-(4-(trifluoromethyl)phenyl)- 4,5dihydro- $1 \mathrm{H}$-pyrazole $(2 \mathrm{~h})$

Yellow powder, yield $75 \%, \mathrm{mp} 145.9-146.3{ }^{\circ} \mathrm{C}$. IR $(\mathrm{KBr}) \vee 3028,2926,1614,1585,1552,1498,1321,1244$, $1155,1064,835 \mathrm{~cm}^{-1} .{ }^{1} \mathrm{H}$ NMR $\left(400 \mathrm{MHz}, \mathrm{DMSO}-d_{6}\right) \delta$ $2.24\left(\mathrm{~s}, 3 \mathrm{H}, \mathrm{CH}_{3}\right), 3.14(\mathrm{dd}, \mathrm{Jax}=6.61 \mathrm{~Hz}, \mathrm{Jab}=17.57 \mathrm{~Hz}$, $1 \mathrm{H}, \mathrm{Ha}$ ), 3.95 (dd, $J b x=12.24 \mathrm{~Hz}, J a b=17.56 \mathrm{~Hz}, 1 \mathrm{H}, \mathrm{Hb}$ ), $5.50(\mathrm{dd}, \mathrm{Jax}=6.60 \mathrm{~Hz}, \mathrm{Jbx}=12.24 \mathrm{~Hz}, 1 \mathrm{H}, \mathrm{Hx}), 6.71-7.22$ (m, 9H, Ar-H), 7.75 (d, J= 8.4 Hz, 2H, Ar-H), $7.91(\mathrm{~d}, J=$ $8.4 \mathrm{~Hz}, 2 \mathrm{H}, \mathrm{Ar}-\mathrm{H}$ ). Anal. Calcd for $\mathrm{C}_{23} \mathrm{H}_{19} \mathrm{~F}_{3} \mathrm{~N}_{2}$ : C, 72.62; H, 5.03; N, 7.36. Found: C, 72.47; H, 4.89; N, 7.56.

1-Phenyl-5-(thiophen-2-yl)-3-(4-(trifluoromethyl)phenyl)-4,5-dihydro-1 $H$-pyrazole (2i)

Orange powder, yield $65 \%$, mp $127.0-127.5{ }^{\circ} \mathrm{C}$. IR $(\mathrm{KBr}) \vee 3097,1618,1591,1529,1479,1315,1220,1166$, 1064, $825 \mathrm{~cm}^{-1} .{ }^{1} \mathrm{H}$ NMR $\left(300 \mathrm{MHz}\right.$, DMSO- $\left.d_{6}\right) \delta 3.25$ (dd, Jax = 6.56 Hz, Jab = 17.61 Hz, 1H, Ha), 3.93 (dd, Jbx = $12.33 \mathrm{~Hz}, J a b=17.61 \mathrm{~Hz}, 1 \mathrm{H}, \mathrm{Hb}), 5.96(\mathrm{dd}, J a x=6.57 \mathrm{~Hz}$, $J b x=12.33 \mathrm{~Hz}, 1 \mathrm{H}, \mathrm{Hx}), 6.77-7.38(\mathrm{~m}, 8 \mathrm{H}, \mathrm{Ar}-\mathrm{H}), 7.76(\mathrm{~d}$, $J=8.1 \mathrm{~Hz}, 2 \mathrm{H}, \mathrm{Ar}-\mathrm{H}), 7.96(\mathrm{~d}, J=8.1 \mathrm{~Hz}, 2 \mathrm{H}, \mathrm{Ar}-\mathrm{H})$. Anal. Calcd for $\mathrm{C}_{20} \mathrm{H}_{15} \mathrm{~F}_{3} \mathrm{~N}_{2} \mathrm{~S}$ : C, 64.50; H, 4.06; N, 7.52. Found: C, 64.14; H, 4.13; N, 7.47.

$N, N$-Dimethyl-4-(1-phenyl-3-(4-(trifluoromethyl)phenyl)-4,5-dihydro- $1 \mathrm{H}$-pyrazol-5-yl)aniline (2j)

Yellow powder, yield $77 \%, \mathrm{mp} 128.8-129.4{ }^{\circ} \mathrm{C}$. IR $(\mathrm{KBr})$ v 3070, 2955, 2845, 1614, 1587, 1519, 1492, 1320, $1240,1163,1055,806 \mathrm{~cm}^{-1} .{ }^{1} \mathrm{H}$ NMR $(400 \mathrm{MHz}, \mathrm{DM}-$ SO- $\left.d_{6}\right) \delta 2.81\left(\mathrm{~s}, 6 \mathrm{H}, 2 \mathrm{CH}_{3}\right), 3.10(\mathrm{dd}, J a x=6.35 \mathrm{~Hz}, J a b=$ $17.46 \mathrm{~Hz}, 1 \mathrm{H}, \mathrm{Ha}$ ), $3.86(\mathrm{dd}, J b x=12.33 \mathrm{~Hz}, J a b=17.47$ $\mathrm{Hz}, 1 \mathrm{H}, \mathrm{Hb}), 5.44(\mathrm{dd}, J a x=6.32 \mathrm{~Hz}, J b x=12.31 \mathrm{~Hz}, 1 \mathrm{H}$, $\mathrm{Hx}), 6.63-7.17(\mathrm{~m}, 9 \mathrm{H}, \mathrm{Ar}-\mathrm{H}), 7.72(\mathrm{~d}, J=8.4 \mathrm{~Hz}, 2 \mathrm{H}, \mathrm{Ar}-$ $\mathrm{H}), 7.91(\mathrm{~d}, J=8.4 \mathrm{~Hz}, 2 \mathrm{H}, \mathrm{Ar}-\mathrm{H})$. Anal. Calcd for $\mathrm{C}_{24} \mathrm{H}-$ ${ }_{22} \mathrm{~F}_{3} \mathrm{~N}_{3}$ : C, 70.40; H, 5.42; N, 10.26. Found: C, 70.56; H, $5.35 ; \mathrm{N}, 10.43$.

5-(Naphthalen-1-yl)-1-phenyl-3-(4-(trifluoromethyl) phenyl)-4,5-dihydro-1H-pyrazole (2k)

Orange powder, yield $79 \%, \mathrm{mp} 94.4-94.6{ }^{\circ} \mathrm{C}$. IR $(\mathrm{KBr}) \vee 3059,1635,1591,1533,1483,1321,1259,1168$, 1064, $835 \mathrm{~cm}^{-1} .{ }^{1} \mathrm{H}$ NMR (400 MHz, DMSO- $\left.d_{6}\right) \delta 3.12$ $(\mathrm{dd}, J a x=6.56 \mathrm{~Hz}, J a b=17.40 \mathrm{~Hz}, 1 \mathrm{H}, \mathrm{Ha}), 4.01(\mathrm{dd}, J b x=$ $12.81 \mathrm{~Hz}, J a b=17.39 \mathrm{~Hz}, 1 \mathrm{H}, \mathrm{Hb}), 6.26$ (dd, Jax $=6.56 \mathrm{~Hz}$,
$J b x=12.80 \mathrm{~Hz}, 1 \mathrm{H}, \mathrm{Hx}), 6.71-8.00(\mathrm{~m}, 16 \mathrm{H}, \mathrm{Ar}-\mathrm{H})$. Anal. Calcd for $\mathrm{C}_{26} \mathrm{H}_{19} \mathrm{~F}_{3} \mathrm{~N}_{2}: \mathrm{C}, 74.99 ; \mathrm{H}, 4.60 ; \mathrm{N}, 6.73$. Found: $\mathrm{C}$, 75.43; H, 4.49; N, 6.56 .

\section{2. Biological Studies}

\section{2. 1. Antioxidant Activity}

\section{2. 1. 1. DPPH Radical Scavenging Activity}

Free radical scavenging capacity of chalcone and pyrazoline derivatives was carried out according to the methods described previously. ${ }^{26,27}$ Briefly, $1 \mathrm{mg}$ of the compound was dissolved in DMSO and four different concentrations $(0.250-0.048 \mu \mathrm{g} / \mathrm{mL}$, approximately) were prepared. $190 \mu \mathrm{L}$ methanol solution of DPPH $(0.1 \mathrm{mM})$ was added on this mixture in a well of 96-well plate. The mixture was allowed to stand in the dark at room temperature for $30 \mathrm{~min}$. Absorbance readings were carried out at 517 $\mathrm{nm}$. The DPPH stock solution without compounds was taken as the negative control. The percentage of inhibition was calculated according to the following:

DPPH scavenging effect $(\%)=\frac{A_{\text {control }}-A_{\text {compound }}}{A_{\text {control }}} \cdot 100$

$A_{\text {control: }}$ Absorbance of the control (containing all reagents except the synthesized compounds).

$A_{\text {compound: }}$ Absorbance of the synthesized compounds.

Tests were repeated in quadruplicate. Ascorbic acid was used as the positive control.

\section{2. 1. 2. ABTS Radical Scavenging Activity}

ABTS radical cations were prepared by dissolving 7 $\mathrm{mM} \mathrm{ABTS}$ and $4.9 \mathrm{mM}$ potassium persulfate, allowing them to react for $16 \mathrm{~h}$ at room temperature in the dark. Then, the ABTS radical solution was diluted with $96 \%$ ethanol to an absorbance recorded at $734 \mathrm{~nm}$. Four different concentrations of the analyzed compounds were prepared according to the above method, DPPH radical scavenging activity. ${ }^{27}$ Absorbance readings were recorded at $734 \mathrm{~nm}$. The percentage of inhibition was calculated according to the following:

ABTS scavenging effect $(\%)=\frac{A_{\text {control }}-A_{\text {compound }}}{A_{\text {control }}} \cdot 100$

Tests were repeated in quadruplicate. Trolox was used as the positive control.

\section{2. 2. Anti-Inflammatory Activity}

The anti-lipoxygenase activity was evaluated as described by Phosrithong and Nuchtavorn with slight modifications described by Yildırım et al. ${ }^{28,29}$ Four different concentrations of chalcone and pyrazoline derivatives 
were added to $250 \mu \mathrm{L}$ of $0.1 \mathrm{M}$ borate buffer $\mathrm{pH} 9.0$, followed by the addition of $250 \mu \mathrm{L}$ of type V soybean lipoxygenase solution in a buffer $(20.000 \mathrm{U} / \mathrm{mL})$. The mixture was incubated at $25^{\circ} \mathrm{C}$ for $5 \mathrm{~min}$ and $1000 \mu \mathrm{L}$ of $0.6 \mathrm{mM}$ linoleic acid solution was added, mixed well and the change in absorbance at $234 \mathrm{~nm}$ was measured for $6 \mathrm{~min}$. The percentage of inhibition was calculated from the following equation:

$$
\% \text { inhibition }=\frac{A_{\text {control }}-A_{\text {compound }}}{A_{\text {control }}} \cdot 100
$$

Tests were repeated in quadruplicate. Indomethacin was used as the positive control. The $\mathrm{IC}_{50}$ values were determined as the concentration of the synthesized compounds required to inhibit lipoxygenase enzyme activity by $50 \%$.

\section{2. 3. Antidiabetic Activity}

\section{2. 3. 1. a-Glucosidase Inhibitory Assay}

The $\alpha$-glucosidase inhibitor activity was evaluated as described by Ramakrishna et al. with slight modifications described by Sen et al. ${ }^{30,31} 40 \mu \mathrm{L}$ of $0.1 \mathrm{M}$ sodium phosphate buffer ( $\mathrm{pH} 6.8$ ) was mixed with $10 \mu \mathrm{L}$ of the synthesized compound at $37^{\circ} \mathrm{C}$. The mixtures were incubated at $25^{\circ} \mathrm{C}$ for 10 minutes with $100 \mu \mathrm{L}$ of $\alpha$-glucosidase which was obtained from Saccharomyces cerevisiae. Then, $50 \mu \mathrm{L}$ of $5 \mathrm{mM}$ p-nitrophenyl- $\alpha$-D-glucopyranoside (pNPG) which was prepared in the buffer, was added to the mixture. The resulting solution was incubated at $25^{\circ} \mathrm{C}$ for 5 minutes again, and absorbance was recorded before and after incubation at $405 \mathrm{~nm}$.

The percentage of inhibition was calculated from the following equation:

$$
\% \text { inhibition }=\frac{A_{\text {control }}-A_{\text {compound }}}{A_{\text {control }}} \cdot 100
$$

Tests were repeated in quadruplicate. Acarbose was used as the positive control. The $\mathrm{IC}_{50}$ values were deter- mined as the concentration of the synthesized compounds required to inhibit $\alpha$-glucosidase enzyme activity by $50 \%$.

\section{2. 4. Statistical Analysis}

The data were given as means \pm standard deviations and analyzed by one-way analysis of variance (ANOVA) followed by the Tukey's multiple comparison tests using GraphPad Prism 5. Differences between means at $p<0.05$ level were considered significant.

\section{Results and Discussion}

\section{1. Chemistry}

Pyrazoline derivatives were synthesized as depicted in Figure 2. Firstly, chalcone derivatives were obtained from 4'-(trifluoromethyl)acetophenone according to Claisen-Schmidt condensation reaction. Then, 2-pyrazoline derivatives were obtained from synthesized chalcones by refluxing in the presence of acidic medium (Figure 2). The structures of the compounds were confirmed by IR, ${ }^{1} \mathrm{H}$ NMR spectroscopic methods and elemental analysis. Physicochemical and spectroscopic characterization of the chalcone derivatives $\mathbf{1} \mathbf{e}, \mathbf{f}, \mathbf{g}, \mathbf{i}, \mathbf{j}$ have been previously described. ${ }^{21-25}$

IR spectra of pyrazoline derivatives afforded aromatic $\mathrm{C}-\mathrm{H}\left(3097-3007 \mathrm{~cm}^{-1}\right)$ stretching, pyrazoline $\mathrm{C}=\mathrm{N}$ stretching $\left(1635-1614 \mathrm{~cm}^{-1}\right)$ and C-F stretching (1168$1118 \mathrm{~cm}^{-1}$ ) bands. When ${ }^{1} \mathrm{H}$ NMR spectra of pyrazoline derivatives were examined, three different characteristic signals belonging to the methylene group at position 4 (Ha and $\mathrm{Hb}$ ) and the methine group at position $5(\mathrm{Hx})$ of the pyrazoline ring were determined. These signals appeared as doublet of doublets due to $\mathrm{ABX}$ spin system in the structures (Figure 3). The $\mathrm{Ha}, \mathrm{Hb}$ and $\mathrm{Hx}$ protons resonated at $3.04-3.25 \mathrm{ppm}\left(J_{\mathrm{ab}}=17.28-17.91 \mathrm{~Hz}\right), 3.86-4.02 \mathrm{ppm}\left(J_{\mathrm{ax}}\right.$ $=6.12-6.66 \mathrm{~Hz})$ and $5.44-6.26 \mathrm{ppm}\left(J_{\mathrm{bx}}=12.24-13.96\right.$ $\mathrm{Hz}$ ), respectively. Aromatic $\mathrm{CH}$ protons were seen at 6.638.00 as multiplet. Especially ortho protons belonging to ar-<smiles>CC(C)CC(=O)CC(C)C(=O)NC=CC(=O)c1ccc(C(F)(F)F)cc1</smiles>

1a and 2a: $\mathrm{Ar}=2,6$-dimethylphenyl, $1 \mathbf{b}$ and $\mathbf{2 b}: \mathrm{Ar}=2,6$-dichlorophenyl, 1c and 2c: $\mathrm{Ar}=2$-methylphenyl,

1d and 2d: $\mathrm{Ar}=4$-isopropylphenyl,

1e and 2e: $\mathrm{Ar}=$ phenyl,

1f and 2f: $\mathrm{Ar}=4$-chloro,
$1 \mathrm{~g}$ and 2g: $\mathrm{Ar}=4-$ methylphenyl,

$1 \mathbf{h}$ and $2 \mathrm{~h}: \mathrm{Ar}=3$-methylphenyl

$1 \mathrm{i}$ and 2i: $\mathrm{Ar}=$ thiophen-2-yl,

1j and $\mathbf{2 j}$ : $\mathrm{Ar}=4$-dimethylaminophenyl,

$1 \mathbf{k}$ and 2k: Ar=naphthalen-1-yl

Figure 2. The synthetic pathway for compounds 1a-1k and 2a-2k. Reagents and conditions: (i) methanol, NaOH, 10 h; (ii) phenylhydrazine hydrochloride, acetic acid, reflux, $12 \mathrm{~h}$. 


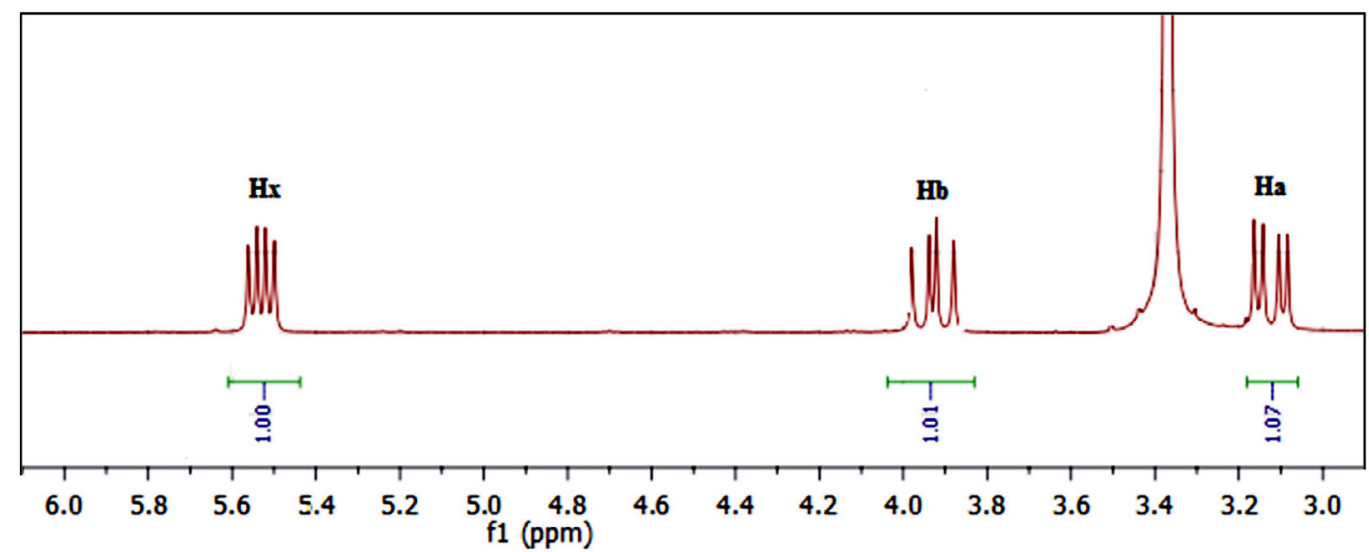

Figure 3. Esxample ${ }^{1} \mathrm{H}$ NMR spectrum of pyrazoline protons ( $\mathrm{Ha}, \mathrm{Hb}$ and $\left.\mathrm{Hx}\right)$.

omatic ring (bearing trifluoromethyl substituent) were observed as doublet $(J=8.1-8.4 \mathrm{~Hz})$ at $7.72-7.96 \mathrm{ppm}$.

\section{2. Biological Evaluation}

All chalcone and pyrazoline derivatives were screened for in vitro antioxidant activity with DPPH and
ABTS assays, anti-inflammatory activity with lipoxygenase (LOX) inhibition assay and antidiabetic activity with a-glucosidase inhibition assay. All $\mathrm{IC}_{50}$ values of compounds against reference standards were given as Table 1. Results demonstrated that all pyrazoline derivatives had a very strong anti-inflammatory activity with $\mathrm{IC}_{50}$ values between $0.68 \pm 0.07$ and $4.45 \pm 0.25 \mu \mathrm{M}$ when compared to

Table 1. Antioxidant, anti-inflammatory and antidiabetic activities of synthesized compounds*

\begin{tabular}{|c|c|c|c|c|}
\hline \multirow{3}{*}{ Compound } & \multicolumn{3}{|c|}{$\mathrm{IC}_{50}(\mu \mathrm{M})$} & \multirow{3}{*}{$\begin{array}{c}\text { Antidiabetic } \\
\text { activity } \\
\text { a-glucosidase } \\
\text { inhibitory activity }\end{array}$} \\
\hline & \multicolumn{2}{|c|}{$\begin{array}{c}\text { Antioxidant } \\
\text { activity }\end{array}$} & $\begin{array}{c}\text { Anti-inflammatory } \\
\text { activity }\end{array}$ & \\
\hline & DPPH activity & ABTS activity & $\begin{array}{l}\text { Anti-lipoxgenase } \\
\text { activity }\end{array}$ & \\
\hline 1a & $>1000$ & $>1000$ & $128.40 \pm 3.92^{\mathrm{f}}$ & $381.20 \pm 9.29^{\mathrm{c}, \mathrm{d}}$ \\
\hline $1 b$ & $>1000$ & $852.80 \pm 51.42^{c}$ & $48.47 \pm 2.29^{c}$ & $460.50 \pm 3.89^{\mathrm{d}, \mathrm{e}}$ \\
\hline $1 \mathrm{c}$ & $211.60 \pm 3.48^{\mathrm{b}}$ & $869.00 \pm 64.4^{c}$ & $121.50 \pm 4.77^{\mathrm{f}}$ & $746.10 \pm 20.95^{\mathrm{h}, \mathrm{i}, \mathrm{j}}$ \\
\hline 1d & $389.50 \pm 2.22^{\mathrm{c}, \mathrm{d}, \mathrm{e}, \mathrm{f}}$ & $807.00 \pm 16.88^{c}$ & $79.35 \pm 0.93^{\mathrm{d}}$ & $338.60 \pm 29.32^{c}$ \\
\hline 1e & $282.10 \pm 15.92^{\mathrm{b}, \mathrm{c}}$ & $>1000$ & $304.60 \pm 1.61^{\mathrm{h}}$ & $>1000$ \\
\hline 1f & $>1000$ & $>1000$ & $50.97 \pm 2.75^{c}$ & $377.40 \pm 4.35^{\mathrm{c}, \mathrm{d}}$ \\
\hline $1 \mathrm{~g}$ & $286.10 \pm 19.48^{\mathrm{b}, \mathrm{c}}$ & $635.80 \pm 11.45^{\mathrm{b}, \mathrm{c}}$ & $105.90 \pm 0.02^{\mathrm{e}}$ & $>1000$ \\
\hline $1 \mathrm{~h}$ & $321.30 \pm 9.96^{\mathrm{b}, \mathrm{c}}$ & $>1000$ & $38.42 \pm 3.02^{\mathrm{b}}$ & $>1000$ \\
\hline $1 \mathrm{i}$ & $>1000$ & $907.40 \pm 26.80^{c}$ & $85.13 \pm 1.56^{\mathrm{d}}$ & $>1000$ \\
\hline $1 \mathrm{j}$ & $494.00 \pm 10.41^{\mathrm{e}, \mathrm{f}, \mathrm{g}, \mathrm{h}, \mathrm{i}}$ & $204.40 \pm 4.29^{\mathrm{a}, \mathrm{b}}$ & $193.60 \pm 0.33 \mathrm{~g}$ & $>1000$ \\
\hline $1 \mathbf{k}$ & $>1000$ & $>1000$ & $83.07 \pm 2.31^{\mathrm{d}}$ & $823.8 \pm 0.43^{j}$ \\
\hline $2 a$ & $534.40 \pm 5.02^{\mathrm{h}, \mathrm{i}, \mathrm{j}}$ & $136.30 \pm 3.97^{\mathrm{a}}$ & $0.68 \pm 0.07^{\mathrm{a}}$ & $81.09 \pm 0.70^{\mathrm{a}, \mathrm{b}}$ \\
\hline $2 b$ & $586.10 \pm 44.19^{\mathrm{i}, \mathrm{j}}$ & $117.40 \pm 0.18^{\mathrm{a}}$ & $0.76 \pm 0.09^{\mathrm{a}}$ & $463.40 \pm 6.50^{\mathrm{d}, \mathrm{e}}$ \\
\hline $2 c$ & $505.80 \pm 23.04^{\mathrm{f}, \mathrm{g}, \mathrm{h}, \mathrm{i}}$ & $127.00 \pm 0.90^{\mathrm{a}}$ & $1.56 \pm 0.08^{\mathrm{a}}$ & $613.80 \pm 14.13^{\mathrm{f}, \mathrm{g}}$ \\
\hline $2 d$ & $342.90 \pm 3.29^{b, c, d}$ & $108.00 \pm 2.92^{\mathrm{a}}$ & $2.15 \pm 0.13^{\mathrm{a}}$ & $596.90 \pm 18.00^{\mathrm{f}, \mathrm{g}}$ \\
\hline $2 e$ & $645.00 \pm 22.0^{j}$ & $130.10 \pm 2.00^{\mathrm{a}}$ & $1.73 \pm 0.04^{\mathrm{a}}$ & $519.50 \pm 18.72^{\mathrm{e}, \mathrm{f}}$ \\
\hline $2 f$ & $524.20 \pm 20.82^{\mathrm{g}, \mathrm{h}, \mathrm{i}, \mathrm{j}}$ & $92.62 \pm 5.45^{\mathrm{a}}$ & $3.11 \pm 0.05^{\mathrm{a}}$ & $451.30 \pm 13.76^{\mathrm{c}, \mathrm{d}, \mathrm{e}}$ \\
\hline $2 \mathrm{~g}$ & $898.90 \pm 5.76^{\mathrm{k}}$ & $112.20 \pm 2.12^{\mathrm{a}}$ & $4.45 \pm 0.25^{\mathrm{a}}$ & $637.90 \pm 1.67^{\mathrm{f,g}}$ \\
\hline $2 \mathrm{~h}$ & $>1000$ & $150.90 \pm 0.57^{\mathrm{a}}$ & $2.14 \pm 0.11^{\mathrm{a}}$ & $657.30 \pm 14.68^{g, h}$ \\
\hline $2 \mathbf{i}$ & $855.60 \pm 19.93^{\mathrm{k}}$ & $136.40 \pm 0.15^{\mathrm{a}}$ & $4.41 \pm 0.02^{\mathrm{a}}$ & $812.70 \pm 5.13^{\mathrm{i}, \mathrm{j}}$ \\
\hline $2 j$ & $>1000$ & $57.13 \pm 0.03^{\mathrm{a}}$ & $1.19 \pm 0.05^{\mathrm{a}}$ & $201.10 \pm 5.22^{\mathrm{b}}$ \\
\hline $2 \mathbf{k}$ & $464.80 \pm 11.38^{\mathrm{d}, \mathrm{e}, \mathrm{f}, \mathrm{g}}$ & $94.24 \pm 0.86^{\mathrm{a}}$ & $1.22 \pm 0.07^{\mathrm{a}}$ & $417.60 \pm 10.53^{\mathrm{c}, \mathrm{d}, \mathrm{e}}$ \\
\hline
\end{tabular}

Ascorbic acid

Trolox

$14.56 \pm 0.60^{\mathrm{a}}$

$12.67 \pm 0.28^{\mathrm{a}}$

$50.45 \pm 0.20^{c}$ Acarbose

\footnotetext{
${ }^{\star}$ Each value in the table is represented as mean $\pm \mathrm{SD}(n=4)$. Different letter superscripts in the same column indicate significant differences $(p<0.05)$.
} 


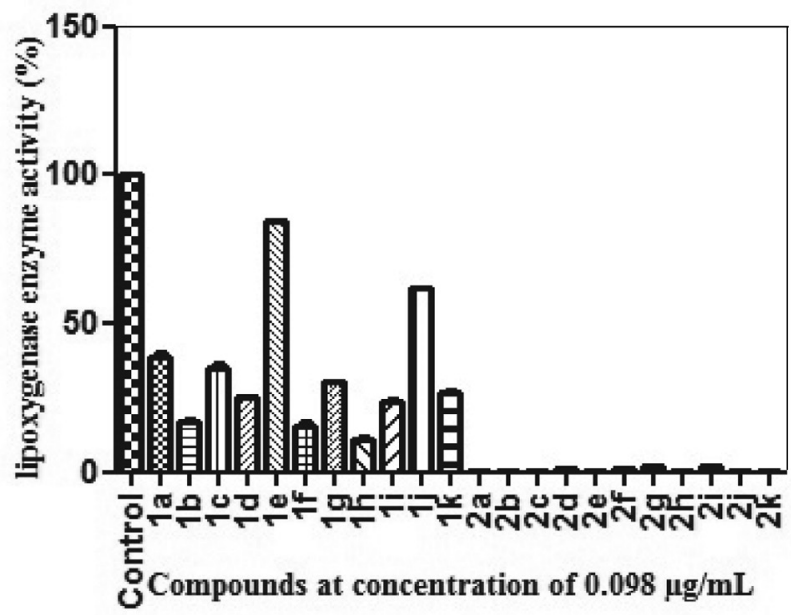

Figure 4. Anti-lipoxygenase activity of chalcone and pyrazoline derivatives.

reference standard indomethacin $\left(\mathrm{IC}_{50}=50.45 \pm 0.20\right.$ $\mu \mathrm{M})$. Additionally, compound 2a inhibited 5-lipoxygenase activity by $99.73 \%$ at a concentration of $0.098 \mu \mathrm{g} / \mathrm{mL}$. Chalcone derivatives $\mathbf{1 a}-\mathbf{k}$ at a concentration of $0.098 \mu \mathrm{g} /$ $\mathrm{mL}$ inhibited lipoxygenase enzyme activity with the inhibition rate of $88.85-15.83 \%$ compared to the control (Figure 4).

Synthesized compounds exhibited low antioxidant activity. Only compound $\mathbf{2 j}$ showed the best antioxidant activity according to ABTS assay with $\mathrm{IC}_{50}$ value of $57.13 \pm$ $0.03 \mu \mathrm{M}$ compared to the reference standard trolox (12.67 $\pm 0.28 \mu \mathrm{M})$.

Antidiabetic activity of all compounds was evaluated by the $\alpha$-glucosidase inhibition assay. These results showed that compound 2a showed maximum a-glucosidase inhibitory activity with the $\mathrm{IC}_{50}$ value of $81.09 \pm 0.70 \mu \mathrm{M}\left(\mathrm{IC}_{50}\right.$ for acarbose $62.04 \pm 3.32 \mu \mathrm{M}$ ).

\section{3. ADME Calculations}

The prediction of ADME properties of the compounds is a very important development of new drug candidates. Therefore the druglike molecule was carried out by using the Lipinski rule of five and Veber rule. ${ }^{32-34} \mathrm{Cal}-$ culations were performed using molinspiration online server. The screening results were presented in Table 2.

The molecular weights varied from 276.25 to 435.27 for the synthesized compounds which are lower than the maximum molecular weight of 500. All the compounds are having $\log \mathrm{P}$ in the range of 3.48-6.22. The number of hydrogen bond acceptors of all compounds is 4 which is less than the maximum value of ten. On the other hand, all the compounds have zero hydrogen bond donors which must be less than the maximum value of five. Furthermore, the number of rotatable bonds is in the range of $4-5$ which is lower than the maximum value of 10 . Similarly the polar

Table 2. Predicted ADME, Lipinski and Veber parameters of the synthesized compounds.

\begin{tabular}{ccccccc}
\hline \multirow{2}{*}{ Compound } & \multicolumn{3}{c}{ Lipinski rule of five } & & \multicolumn{2}{c}{ Veber rule } \\
& MW & Log P & $\boldsymbol{n}$-ON & $\boldsymbol{n}$-OHNH & $\boldsymbol{n}$-ROTB & TPSA \\
\hline $\mathbf{1 a}$ & 304.31 & 4.78 & 4 & 0 & 4 & 17.07 \\
$\mathbf{1 b}$ & 345.14 & 5.32 & 4 & 0 & 4 & 17.07 \\
$\mathbf{1 c}$ & 290.28 & 4.55 & 4 & 0 & 4 & 17.07 \\
$\mathbf{1 d}$ & 318.33 & 5.01 & 4 & 0 & 5 & 17.07 \\
$\mathbf{1 e}$ & 276.25 & 4.32 & 4 & 0 & 4 & 17.07 \\
$\mathbf{1 f}$ & 310.70 & 4.82 & 4 & 0 & 4 & 17.07 \\
$\mathbf{1 g}$ & 290.28 & 4.55 & 4 & 0 & 4 & 17.07 \\
$\mathbf{1 h}$ & 290.28 & 4.55 & 4 & 0 & 4 & 17.07 \\
$\mathbf{1 i}$ & 282.28 & 3.48 & 4 & 0 & 4 & 45.31 \\
$\mathbf{1 j}$ & 319.32 & 4.12 & 4 & 0 & 5 & 20.31 \\
$\mathbf{1 k}$ & 326.31 & 5.04 & 4 & 0 & 4 & 17.07 \\
$\mathbf{2 a}$ & 394.43 & 5.69 & 4 & 0 & 4 & 15.60 \\
$\mathbf{2 b}$ & 435.27 & 6.22 & 4 & 0 & 4 & 15.60 \\
$\mathbf{2 c}$ & 380.41 & 5.48 & 4 & 0 & 5 & 15.60 \\
$\mathbf{2 d}$ & 408.46 & 5.89 & 4 & 0 & 4 & 15.60 \\
$\mathbf{2 e}$ & 366.38 & 5.27 & 4 & 0 & 4 & 15.60 \\
$\mathbf{2 f}$ & 400.82 & 5.75 & 4 & 0 & 4 & 15.60 \\
$\mathbf{2 g}$ & 380.41 & 5.48 & 4 & 0 & 4 & 15.60 \\
$\mathbf{2 h}$ & 380.41 & 5.48 & 4 & 0 & 4 & 43.84 \\
$\mathbf{2 i}$ & 372.41 & 4.88 & 4 & 0 & 5 & 18.84 \\
$\mathbf{2 j}$ & 409.45 & 5.07 & 4 & 0 & 4 & 15.60 \\
$\mathbf{2 k}$ & 416.44 & 5.91 & 4 & 0 & & 4 \\
\hline
\end{tabular}

TPSA: Topological polar surface area, $n$-ON: number of hydrogen bond acceptors, $n$-OHNH: number of hydrogen bond donors, $n$-ROTB: number of rotatable bonds. Calculations were performed using Molinspiration online property calculation toolkit (http://www.molinspiration.com). 
surface area of all synthesized compounds is indicated to be in the range of $15.60-45.31 \AA^{2}$ which is less than the maximum value of $140 \AA^{2}$. These values demonstrate that none of the synthesized compounds are violating the Lipinski and Veber rules.

\section{Conclusion}

New pyrazoline derivatives were synthesized from chalcone derivatives and the designed molecules were investigated for their drug-likeness properties which were defined as Lipinski and Veber rules. All compounds were tested for their antioxidant (DPPH and ABTS), anti-lipoxygenase and $\alpha$-glucosidase inhibitory activities. These results showed that pyrazoline derivatives exhibited better activity than chalcone derivatives. Especially pyrazoline derivatives $\mathbf{2 a}-\mathbf{k}$ showed very strong anti-lipoxygenase activity each with greater activity than reference drug indomethacin. Also, compounds $\mathbf{2} \mathbf{j}$ and $\mathbf{2 a}$ demonstrated good antioxidant and $\alpha$-glucosidase inhibitory activity, respectively. These findings revealed that the pyrazoline core could lead to considerably active molecules.

\section{Conflict of Interest}

Authors declare no conflict of interest.

\section{References}

1. M. Asad, M. N. Arshad, S. A. Khan, M. Oves, M. Khalid, A. M. Asiri, A. A. C. Braga, J. Mol. Struct. 2020, 1201, 1-14.

DOI:10.1016/j.molstruc.2019.127186

2. M. R. Bhosle, A. R. Deshmukh, S. Pal, A. K. Srivastava, R. A. Mane, Bioorg. Med. Chem. Lett. 2015, 25, 2442-2446.

DOI:10.1016/j.bmcl.2015.03.068

3. C. Kharbanda, M. S. Alam, H. Hamid, K. Javed, S. Shafi, Y. Ali, P. Alam, M. A. Q. Pasha, A. Dhulap, S. Bano, S. Nazreen, S. Haider, Bioorg. Med. Chem. Lett. 2014, 24, 5298-5303.

DOI:10.1016/j.bmcl.2014.09.044

4. D. S. Raghuvanshi, N. Verma, S. V. Singh, S. Khare, A. Pal, A. S. Negi, Bioorg. Chem. 2019, 88, 1-11.

DOI:10.1016/j.bioorg.2019.102933

5. G. Turan-Zitouni, P. Chevallet, F. S. Kılıç, K. Erol, Eur. J. Med. Chem. 2000, 35, 635-641.

DOI:10.1016/S0223-5234(00)00152-5

6. C. Kharbanda, M. S. Alam, H. Hamid, K. Javed, S. Bano, A. Dhulap, Y. Ali, S. Nazreen, S. Haider, Bioorgan. Med. Chem. 2014, 22, 5804-5812. DOI:10.1016/j.bmc.2014.09.028

7. F. Tok, B. İ. Abas, Ö. Çevik, B. Koçyiğit-Kaymakçığlu, Bioorg. Chem. 2020, 102, 104063.

DOI:10.1016/j.bioorg.2020.104063

8. H. S. ElBordiny, M. M. El-Miligy, S. E. Kassab, H. Daabees, W. A. M. Ali, S. A. M. El-Hawash, Eur. J. Med. Chem. 2018, 145, 594-605. DOI:10.1016/j.ejmech.2018.01.026

9. F. Chaudhry, S. Naureen, R. Huma, A. Shaukat, M. Al-Rashi- da, N. Asif, M. Ashraf, M. A. Munawar, M. A. Khan, Bioorg. Chem. 2017, 71, 102-109.

DOI:10.1016/j.bioorg.2017.01.017

10. M. Sathish, G. Meenakshi, S. Xavier, S. Sebastian, S. Periandy, N. Ahmad, J. Jamalis, M. Rosli, H. Fun, J. Mol. Struct. 2018, 1164, 420-437. DOI:10.1016/j.molstruc.2018.03.004

11. B. Sever, M. D. Altıntop, M. O. Radwan, A. Ozdemir, M. Otsuka, M. Fujita, H. I. Ciftci, Eur. J. Med. Chem. 2019, 182, 1-17. DOI:10.1016/j.ejmech.2019.111648

12. B. Shu, Q. Yu, D. Hu, T. Che, S. Zhang, D. Li, Bioorg. Med. Chem. Lett. 2020, 30, 1-4.

DOI:10.1016/j.bmcl.2019.126925

13. D. Moi, A. Nocentini, A. Deplano, G. Balboni, C. T. Supuran, V. Onnis, Eur. J. Med. Chem. 2019, 182, 1-11.

DOI:10.1016/j.ejmech.2019.111638

14. A. Mumtaz, A. Majeed, S. Zaib, S. Rahman, S. Hameed, A. Saeed, H. Rafique, E. Mughal, A. Maalik, I. Hussain, J. Iqbal, Bioorg. Chem. 2019, 90, 1-9.

DOI:10.1016/j.bioorg.2019.103036

15. N. M. A. Gawad, G. S. Hassan, H. H. Georgey, Med. Chem. Res. 2012, 21, 983-994. DOI:10.1007/s00044-011-9606-4

16. S. Viveka, P. Shama, G. K. Nagaraja, S. Ballav, S. Kerkar, Eur. J. Med. Chem. 2015, 101, 442-451.

DOI:10.1016/j.ejmech.2015.07.002

17. P. Shah, A. D. Westwell, J. Enzym Inhib. Med. Chem. 2007, 22, 527-540. DOI:10.1080/14756360701425014

18. K. Haranahalli, T. Honda, I. Ojima, J. Fluorine Chem. 2017, 217, 29-40. DOI:10.1016/j.jfluchem.2018.11.002

19. N. Beyhan, B. Kocyigit-Kaymakcioglu, S. Gümrü, F. Arıcıoğlu, Arab. J. Chem. 2017, 10, 2073-2081.

DOI:10.1016/j.arabjc.2013.07.037

20. A. Ahmad, A. Husain, S. A. Khan, M. Mujeeb, A. Bhandari, J. Saudi Chem. Soc. 2016, 20, 577-584.

DOI:10.1016/j.jscs.2014.12.004

21. X. Huang, J. Li, X. Li, J. Wang, Y. Peng, G. Song, RSC. Adv. 2019, 9, 26419-26424. DOI:10.1039/C9RA05708K

22. T. T. Nguyen, G. D. Kortman, K. L. Hull, Organometallics, 2016, 35, 1713-1725. DOI:10.1021/acs.organomet.6b00111

23. D. Liu, J. Yu, J. Cheng, Tetrahedron, 2014, 70, 1149-1153. DOI:10.1016/j.tet.2013.12.077

24. W. Katsuaki, G. Takuya, O. Yuichi, S. Katsuhiko, A. Takahisa, A. Wolfram, H. Achim, L. Peter, Preparation of nematicidal pyrazoles, US Patent Number 6310049, date of patent October 30, 2001.

25. R. Shalaby, J. P. Petzer, A. Petzer, U. M. Ashraf, E Atari, F. Alasmari, S. Kumarasamy, Y. Sari, A. Khalil, J. Enzym Inhib. Med. Ch. 2019, 34, 863-876.

DOI:10.1080/14756366.2019.1593158

26. Y. Zou, S. K. C. Chang, Y. Gu, S. Y. Qian, J. Agric. Food Chem. 2011, 59, 2268-2276. DOI:10.1021/jf104640k

27. A. Şen, Marmara Pharm. J. 2018, 22, 328-333. DOI:10.12991/mpj.2018.71

28. A. Yıldırım, A. Şen, A. Doğan, L. Bitiş, Int. J. Sec. Metabolite, 2019, 6, 211-222.

29. N. Phosrithong, N. Nuchtavorn, Eur. J. Integr. Med. 2016, 8, 281-285. DOI:10.1016/j.eujim.2015.10.002 
30. R. Ramakrsihna, D. Sarkar, P. Schwarz, K. Shetty, Ind. Crops Prod. 2017, 107, 509-517.

DOI: $10.1016 /$ j.indcrop.2017.03.033

31. A. Sen, M. Kurkcuoglu, I. Senkardes, L. Bitis, K. H. C. Baser, J. Essent. Oil Bear. Pl. 2019, 22, 1048-1057.

DOI:10.1080/0972060X.2019.1662333
32. R. Matsa, P. Makam, M. Kaushik, S.L. Hoti, T. Kannan, Eur. J. Pharm. Sci. 2019, 137, 1-12. DOI:10.1016/j.ejps.2019.104986

33. C. A. Lipinski, Drug Discov. Today Technol. 2004, 1, 337-341. DOI:10.1016/j.ddtec.2004.11.007

34. D. F. Veber, S. R. Johnson, H. Y. Cheng, B. R. Smith, K. W. Ward, K. D. Kopple, J. Med. Chem. 2002, 45, 2615-2623. DOI:10.1021/jm020017n

\section{Povzetek}

Iz halkonskih derivatov smo sintetizirali serijo 1-fenil-5-substituiranih-3-(4-(trifluorometil)fenil)-4,5-dihidro- $1 H$-pirazolov. Strukture spojin smo določili s pomočjo IR ter ${ }^{1} \mathrm{H}$ NMR spektroskopskih metod in z elementno analizo. Za vse spojine smo določili in vitro antioksidativno aktivnost s pomočjo DPPH in ABTS metod, antiinflamatorno aktivnost s pomočjo metode inhibicije lipooksigenaze ter antidiabetično aktivnost s pomočjo metode inhibicije $\alpha$-glukozidaze. Še zlasti pirazolinski derivati so izkazali visoke antiinflamatorne aktivnosti, celo večje kot referenčna učinkovina indometacin $\left(\mathrm{IC}_{50}: 50.45 \mu \mathrm{M}\right) \mathrm{z} \mathrm{IC}_{50}$ vrednostmi v območju od 0.68 do $4.45 \mu \mathrm{M}$. V nadaljevanju smo določili še ADME lastnosti vseh halkonskih in pirazolinskih derivatov skladno s pravili Lipinskega in Vebra.

Except when otherwise noted, articles in this journal are published under the terms and conditions of the Creative Commons Attribution 4.0 International License 